\title{
ThimbleSense: a fingertip-wearable tactile sensor for grasp analysis
}

\author{
Edoardo Battaglia, Matteo Bianchi, Alessandro Altobelli, Giorgio Grioli, \\ Manuel G. Catalano, Alessandro Serio, Marco Santello and Antonio Bicchi
}

\begin{abstract}
Accurate measurement of contact forces between hand and grasped objects is crucial to study sensorimotor control during grasp and manipulation. In this work we introduce ThimbleSense, a prototype of individual-digit wearable force/torque sensor based on the principle of intrinsic tactile sensing. By exploiting the integration of this approach with an active marker-based motion capture system, the proposed device simultaneously measures absolute position and orientation of the fingertip, which in turn yields measurements of contacts and force components expressed in a global reference frame. The main advantage of this approach with respect to more conventional solutions is its versatility. Specifically, ThimbleSense can be used to study grasping and manipulation of a wide variety of objects, while still retaining complete force/torque measurements. Nevertheless, validation of the proposed device is a necessary step before it can be used for experimental purposes. In this work we present the results of a series of experiments designed to validate the accuracy of ThimbleSense measurements and evaluate the effects of distortion of tactile afferent inputs caused by the device's rigid shells on grasp forces.
\end{abstract}

\section{INTRODUCTION}

G RASPING objects is a mundane, and yet complex, activity and one of the primary actions to explore and interact with the external world. For these reasons it is not surprising that many studies have been devoted to understanding neuroscientific aspects underpinning human control of forces during object grasp. For example in [1] Flanagan et al. highlight the importance of contact events in the control of manipulation tasks and the importance of predictive control mechanisms that are based on knowledge of object properties. Moreover, in [2] Johansson and Flanagan address how the brain uses tactile information in manipulation tasks, discussing in particular the notion that planning and control is centered on mechanical events that mark transitions between consecutive action phases, and that represent sub-goals of the overall task. Finally, in [3] Wolpert et al. review the topic of human motor learning, focusing on the computational mechanisms involved.

Understanding human grasp is also of interest for its applications to the design of robotic hands and prostheses. In [4] Cutkosky and Howe describe and compare the empirical and analytical approach for robotic grasp analysis, with the former focusing on experiments with humans to extract knowledge to help design better artificial hands, and the latter using analytical instruments to model grasp from a theoretical point of view. For both approaches, to achieve an exhaustive description a complete characterization in terms of (i) force vector components and (ii) contact locations

- E.B., A.S. and A.B. authors are within Centro di Ricerca "E. Piaggio", University of Pisa, 56126 Pisa, Italy

- M.B., A.A., G.G., M.G.C. and A.B. authors are within Italian Institute of Technology, 16163 Genova, Italy.

- M.S. is within School of Biological and Health Systems Engineering, Arizona State University, 85280 Tempe (AZ), USA

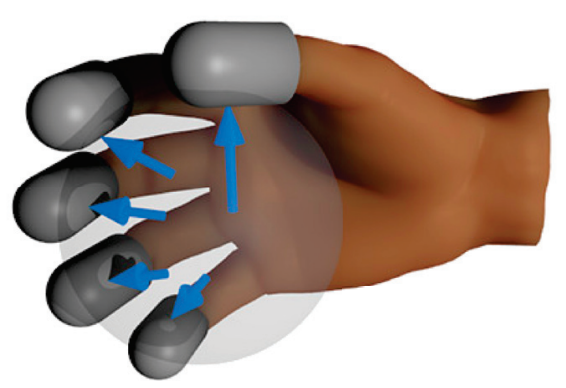

Fig. 1: Concept of the ThimbleSense digit-wearable tactile sensor.

is necessary (see [5] for what concerns the importance of such information in humans and [6] for its relevance for what concerns the mathematical modeling of grasping). Additionally, in ideal conditions the sensing process should be able to minimally constrain the pose of the human hand at contact (iii), to capture the human control aspects with no interference. Finally, preservation of the cutaneous feedback on the fingertips (iv) is also important, since it has been shown that it plays a vital role in grasp force control ([7], [8], [9]). Unfortunately, satisfying all these requirements simultaneously is extremely challenging, and thus technical solutions generally have to resort to a compromise.

A common approach to the problem of measuring forces from the human hand is to build gloves with pressure sensors, which however only provide information about normal forces, and thus do not offer a complete force measurement (i) and can not in general offer a solution for contact point estimation (ii) (see examples reviewed by Dipietro et al. in [10]). More advanced wearable solutions exist such as the one exploiting analysis of the fingernail coloration described by Grieve et al. in [11], which conserves cutaneous cues (iv) but still does not provide torque mea- 


\begin{tabular}{|c|c|c|c|c|c|c|}
\hline & & & Tekscan Grip System & Fingernail sensors & Sensorized objects & ThimbleSense \\
\hline \multirow{3}{*}{ (i) } & \multirow{3}{*}{ Force measurements } & a) Normal Forces & $\checkmark$ & $\checkmark$ & $\checkmark$ & $\checkmark$ \\
\hline & & b) Shear Forces & $x$ & $\checkmark$ & $\checkmark$ & $\checkmark$ \\
\hline & & c) Torques & $x$ & $x$ & $\checkmark$ & $\checkmark$ \\
\hline (ii) & Contacts & & $x$ & $x$ & $\checkmark$ & $\checkmark$ \\
\hline (iii) & Unconstrained grasp & & $\checkmark$ & $\checkmark$ & $x$ & $\checkmark$ \\
\hline (iv) & Cutaneous cues & & $x$ & $\checkmark$ & $\checkmark$ & $x$ \\
\hline
\end{tabular}

TABLE 1: Features of some force measurement devices compared with ThimbleSense.

surements nor contact point estimation. Another approach to the problem of force sensing for human hands consists in building sensorized objects, by assembling parts around one or more force/torque (F/T) sensors, and use them to study grasping and manipulation with a variable number of digits. For example, in [12] Santello and Soechting used a sensorized manipulandum to study force coordination during five-digits grasping, and in [13] a similar device was used to study force coordination during three-digit grasping in an object rotation task. In [14] Zhang et al. used an invert $\mathrm{T}$ sensorized object to investigate sensorimotor memory representation. A collection of sensorized objects can be found in [15], where Zatsiorsky and Latash reviewed the state of art on manipulation analysis.

Force measurement through sensorized objects provides a complete solution for (i). However, it lacks versatility, since it requires building a different object for every task or task condition. Moreover, most objects built this way are not satisfactory for what concerns the unconstrained grasp requirement (iii), since they impose limitations on the way grasping can be performed, e.g., placing sensors at specific locations on the grip devices constraints how subjects grasp the object ([15]). Even when subjects are allowed to choose contact points on grip devices that do not constrain digit placement at predetermined locations (e.g., Fu et al. [16]), this approach is limited by the difficulty of giving a satisfactory measurement of contacts (ii) when more than two digits are used to grasp the object (e.g. $\mathrm{Fu}$ et al. [17]). Additionally, neuroscientific studies often limit the orientation of the sensorized object to a given known configuration (e.g. parallel to the ground) where it is possible to decompose in a straightforward manner grip vs. load force components and test for the achievement of equilibrium conditions (e.g. Baud-Bovy and Soechting [18], even if later developments of the system were used in tilt studies [13], Winges et al. [19]).

In this work we present a sensing approach that was first introduced in a preliminary version in [20], and that for the first time is able to provide a complete characterization of (unconstrained) human and robotic grasp in terms of force measurements and contacts. We propose a fingertip wearable sensor that is able to record all the components of forces exerted on the object (i) and at the same time to provide an estimation of the contact point (ii). As a new contribution with respect to [20], we introduce the integration of a technique to deal with force measurement bias caused by weight (introduced by Atkeson and Hollerbach in [21] and further developed by Kubus et al. in [22]). This, together with the fact that the sensor is wearable, allows the subject to grasp generic objects and to achieve reliable measurements in any grasp orientation, thus solving (iii). Moreover, a major addition to the previous work consists in a quantitative analysis of the accuracy of measurements. In order to be able to achieve such complete measurements we deliberately decided to sacrifice cutaneous feedback (iv) on the fingertips: for this reason in this work an evaluation of the ThimbleSense in experiments with humans was also performed, to evaluate the effects on grip force during a task and define the field of application of the device accordingly.

To better define where ThimbleSense is placed in the scenery of hand force sensing, we report in Table 1 a summary of the devices mentioned in the introduction and of their features, together with the features of ThimbleSense itself.

\section{CONCEPT}

Measurement of forces and torques on a fingertip can be cast as a generic structural mechanics problem. To try and analyze the possible solutions, let us abstract from the physical problem, and consider the simple 2D example shown in (a) No sensor.

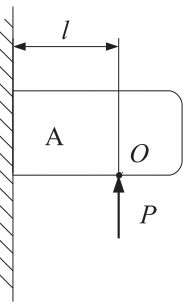

(b) External.

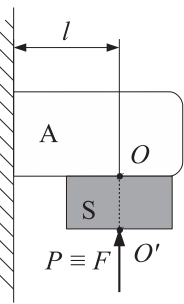

(c) Integrated.

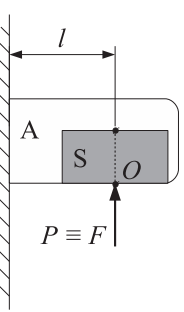

(d) Basal.

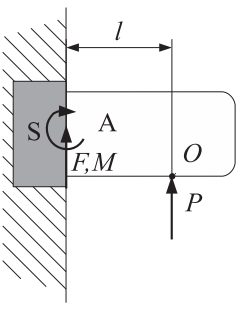

(e) Remote.

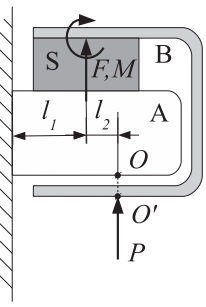

(f) ThimbleSense concept.

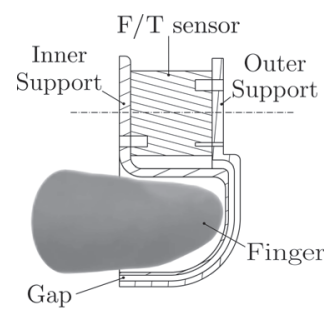

Fig. 2: A basic loaded structure (a), possible ways to sense the load (b-e), and the concept behind our shell-based wearable design (f). 
Fig. 2a. A rigid body $\mathbf{A}$, attached to a frame, withstands a force $P$ applied on a point $O$ at position $l$, perpendicularly with respect to its main axis. Let us suppose that a sensor $\mathbf{S}$, able to measure force $F$ and torque $M$ applied on its surface, is available.

The simplest course of action to measure the applied force is interposing the sensor between the applied force and the object $\mathbf{A}$, as shown in fig. $2 \mathrm{~b}$. This solution, which is in general possible only when the position $l$ is known $a$ priori, has the disavantage of dislocating the point in which the force $P$ is applied from the original $O$ to the remote $O^{\prime}$. This displacement could be recovered by excavating a hole inside the object $\mathbf{A}$ and using it to integrate the sensor (fig. 2c); or it could be removed altogether by splitting the structure in two parts, separating body $\mathbf{A}$ from the frame and interposing the sensor between them, as in fig. $2 \mathrm{~d}$. This would allow, from the measurements of force $F$ and torque $M$, the straightforward reconstruction of $P=F$ and $l=\frac{M}{P}$, and thus of both the magnitude and position of the contact force. It is worth pointing out that without torque measurement it would not be possible to estimate the position of the contact.

The three approaches exposed so far lead to the design of common sensorized objects, but they can not be applied to a human finger: approaches $2 \mathrm{c}$ and $2 \mathrm{~d}$ are invasive with respect to the finger, whereas approach $2 b$ is invasive with respect to the grasp itself, owing to the typical dimensions of force/torque sensors. The problem can then be defined as designing a sensor capable of results similar to those obtainable with approach $2 \mathrm{~d}$ (simultaneous reconstruction of force and contact position), which can be placed on the finger without completely altering the grasp with interposition of a cumbersome object between the finger and the contact. Fig. $2 \mathrm{f}$ shows a possible solution: by assembling the sensor $\mathbf{S}$ between the object $\mathbf{A}$ and a properly designed shell $\mathbf{B}$ we obtain a system which is completely non-invasive to the finger, while also minimizing alteration to the way the load is applied. In this regard it can be noticed that, as in solution $2 \mathrm{~b}$, the load is not directly applied on point $O$ but on a different point $O^{\prime}$; however, unlike solution $2 \mathrm{~b}$, a proper design of the shell $\mathbf{B}$ can substantially reduce the distance $\overline{O O^{\prime}}$. This last solution was selected for our device.

Following this concept, a F/T sensor is assembled between an inner and an outer shell separated by a gap. The finger is placed inside the inner shell, and once the outer shell gets in contact with an object the applied mechanical action is routed though the sensor, which constitutes the only mechanical coupling element between the two shells. This design allows a complete measurement of forces and torques. Specifically, as the geometry of the external support is known, it is possible to obtain the position of the contact centroid of the loading force $P$, through the intrinsic tactile sensing algorithm defined by Bicchi et al. in [23]. More in general, given a surface $S$ with an outward normal defined everywhere, and a distribution $\Delta$ of compressive tractions applied on it, the contact centroid is defined as a point $\mathbf{c}$ such that a wrench exists which is equivalent to $\Delta$ and consists of a force $\mathbf{p}$ directed into $S$ applied to $\mathbf{c}$ and a pure torque $\mathbf{q}$ about the contact normal $\mathbf{n}$. All this quantities can be obtained from the intrinsic tactile algorithm; we refer the interested reader to [23] for more details.
A number of factors must be taken into account to obtain a functional design, namely:

- Size: the device must be as small as possible, to minimize encumbrance. Consequently, all layers between finger and external surface of the outer shell must be as thin as possible. At the same time, the layers need to be thick enough to guarantee a stiffness sufficient to keep the outer shell separated from the inner shell when a load is applied.

- Weight: the device needs to be light, to minimize the effort necessary to carry it. Therefore a material with a high stiffness/weight ratio should be chosen.

- Ergonomics: the device must be shaped in such a way as to leave finger movements unhindered as much as possible.

Overall, the grasping process should ideally be unaffected by wearing the thimbles. If so, it should be possible to seamlessly wear five devices, one on each fingertip, without them excessively interfering with hand movements and grasping capabilities. However, the fact that a rigid shell is placed over the fingertip will necessarily alter grasp control. This issue was studied by Lederman and Klatzky in [24], where it was shown that wearing a rigid shell on the fingertips significantly alters haptic recognition of common objects. A substantial part of the validation procedure presented in this paper will address this issue.

\section{DEsign AND IMPLEMENTATION}

To finalize the mechanical design of the ThimbleSense shells, the ATI nano 17 six axis F/T sensor was selected: the sensors used in all experiments performed for this paper had SI-50-0.5 calibration (see the ATI website [25] for more details and sensor characteristics). Because of its high stiffness/weight ratio, the material chosen to build the thimbles is aluminium. To minimize weight and encumberance of the device, a Finite Element Analysis (FEA) was performed on the CAD model of the device to determine the minimum thickness for the shells and the gap, while still ensuring separation between the shells when they elastically deform under load application. To perform FEA, a load model is needed. From some basic tests performed by pressing a finger on a high precision scale, reasonable bounds of the forces applied were estimated. An average value for the force was $10 \mathrm{~N}$, whereas $35 \mathrm{~N}$ was the higher limit. To be conservative, loads on the structure were modeled as localized forces applied on the bottom of the open ends of either the inner or the outer shell.

A custom material was defined to describe the sensor mechanical properties, with elastic modulus coherent with elastic constants from the data sheet. Trials were performed for various levels of thickness. Here we present the results for a design with $1 \mathrm{~mm}$ thickness for both shells and the gap. Figure 3 shows a static structural model with a localized force applied on the inner shell. It can be seen that the simulation results show deformations smaller than $1 \mathrm{~mm}$. Since the load model chosen is an overestimation of the actual load, this was deemed to be adequate from a mechanical point of view. Figure 4 shows a load model where a force is applied on the outer shell. In this case the force also exhibits 
a significant lateral component. The deformation is close to $1 \mathrm{~mm}$, which is considered to be acceptable owing to the high load.

It is worth noting that it would not be desirable to have a thicker shell, since that would cause greater weight and encumbrance. Conversely, reducing the shells' thickness could cause them to come in contact when a load is applied. The result of our design procedure is shown in Figure 5a, which illustrates an exploded view of the final CAD model, together with the list of components of one ThimbleSense. The final mass of the device is 22 grams, which includes 9 grams from the F/T sensor. Figure 5b shows boundary dimensions of the device.

Grasping objects with a smooth, metal thimble is not an easy task due to the very low friction. To facilitate the task, an artificial fingerpad made of latex and rubber was applied to the outer shell, to mimic the natural frictional properties of human fingerpads (Figure 6a). This cover also allows for some deformation during contact, which increases the contact area: while this is no replacement for the compliance of a fingertip, we found that it made grasping objects easier respect to the making paint solution that was used in [20].

The last element of the thimble design is a reliable way to place it on the fingertips. Ideally, we would like the inner surface of the thimble to be perfectly attached to the skin. To minimize the relative movement between the device and the hand, we used tight finger gloves with extremities covered in velcro loops (Figure 6b), while velcro hooks are applied inside the inner shell portion of the ThimbleSense. This component ensures that the thimbles stay attached to the fingertips and makes the device wearable. Thanks to its compliance, velcro also acts as a soft padding, and helps adapting the thimble to a broader range of finger sizes. Of course this cannot account for all possible size variations: in order to achieve that different sizes need to be provided for the thimbles, which will be done in future work.

\section{MOTION CAPTURE INTEGRATION}

So far we have described a system that allows us to measure generalized forces applied to a thimble, which is worn on fingertips while grasping objects. However, the measurements of the $\mathrm{F} / \mathrm{T}$ sensor are expressed in a frame that is attached to the thimble itself. To locate them in a global reference frame we need to obtain position and orientation of the thimbles. Position and orientation of a rigid body can be estimated from the position of a number of points (a) Model of load.

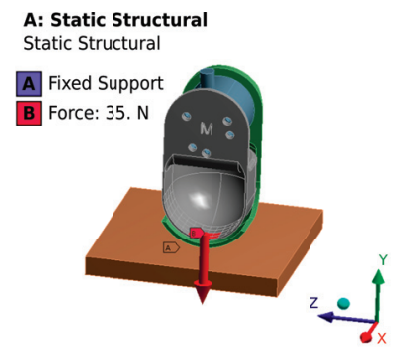

(b) Results

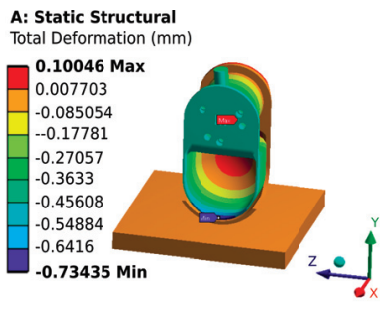

Fig. 3: FEM analysis: load on the inner shell. (a) Model of load.

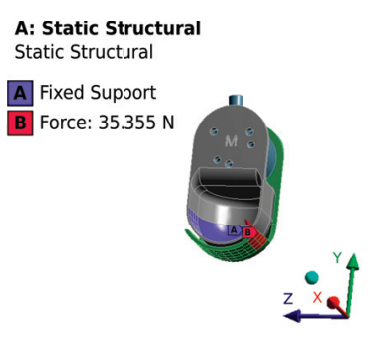

(b) Results.

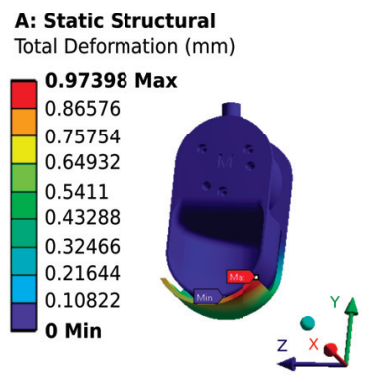

Fig. 4: FEM analysis: load with lateral component on the outer shell. (a) Exploded view.

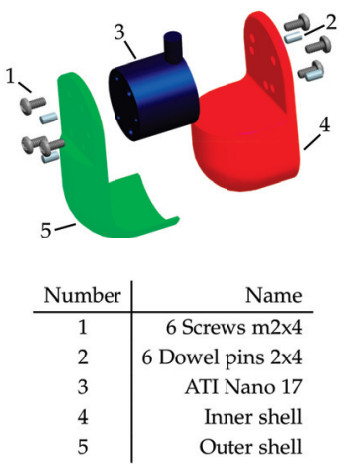

(b) Dimensions.

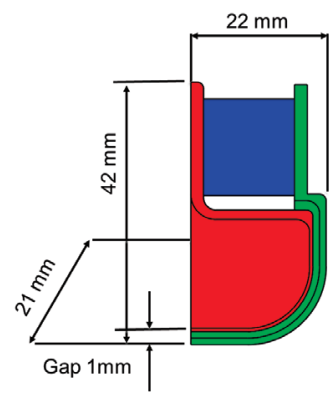

Fig. 5: Shell based wearable device: final design.

attached to it, for example by using the algorithm described by Eggert et al. in [26]. It is worth noting that this algorithm requires at least three non-aligned points to be effective.

Coordinates of points attached to the thimble can be obtained, for example, by using a motion capture system and placing LED markers on a support attached to the thimble. In our setup, we chose the Phase Space motion (a) Artificial finger pad.

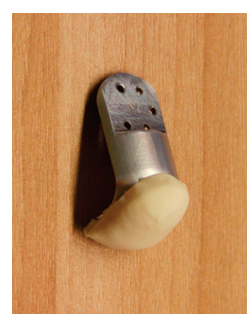

(b) Finger gloves.

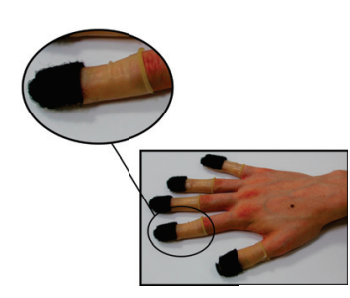

Fig. 6: Setup used to keep thimbles on fingertips. (a) CAD model.

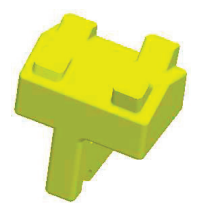

(b) Physical realization.

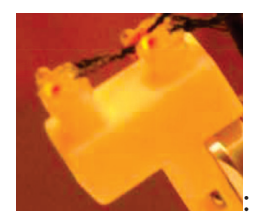

Fig. 7: ABS support for Phase Space markers. 
capture system [27], and designed and realized suitable ABS plastic supports to attach the LED beacons to the thimbles, which are all uniquely identified by the system through an ID. To make sure that the minimum number of three markers needed to estimate rigid body motion is always available, four marker slots were designed on the support, to be more robust to occasional loss of markers during the acquisition. Such slots are all placed at different heights over the surface of the support, to maximize visibility. The CAD model and physical realization are shown in Figure 7. Knowledge of the thimbles orientation allows us to express forces and measurements in a fixed base reference frame $\{B\}$. From now on all quantities will be expressed in $\{B\}$, unless specified.

It is worth pointing out that, while the choice of a motion capture system represented a convenient solution to our problem of estimating position and orientation of ThimbleSense, it is not an intrinsic part of the device. Any solution that can be employed to measure position and orientation of the fingertips could be adapted to replace it: consideration of different methods will be part of future work.

\section{WEIGHT BIAS COMPENSATION}

To avoid measurement errors the F/T sensors need to be zeroed before each acquisition. This static zeroing cannot however take into account the bias that a body attached to the sensor induces with its weight when orientation changes. In this case it is possible to compensate for the weight-induced bias from knowledge of the mass and of the center of mass coordinates of the structure attached. In particular we used the techniques described in [21], [22], applying it on data acquired during a motion for which the ThimbleSense orientation was slowly changed around the whole workspace, with no object being grasped. Under these conditions the dynamic actions can be neglected and the F/T measurements collected can be ascribed only to the weight of the external shell and the ABS support. (a) Thumb force.

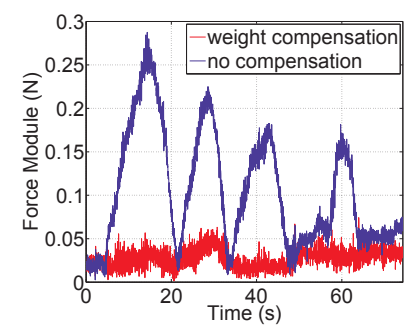

(b) Thumb torque.

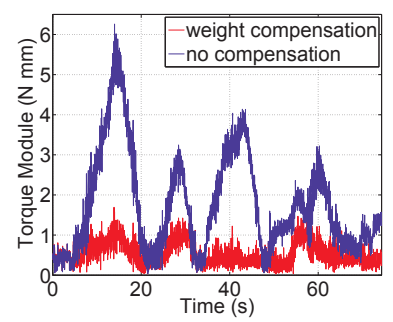

Fig. 8: Measurement errors during calibration with and without bias removal.

Figure 8 shows measurements of the norm of force and torque for the thumb, with and without compensation. It can be seen that the error with respect to the expected zero value is consistently lower when weight compensation is applied. Similar results are observed for the other fingers, as quantified by the Root Mean Square Errors in Table 2. Notice that the error is reduced to approximately one third of the error without compensation.

\begin{tabular}{ccccc}
\hline & \multicolumn{2}{c}{ Force $(\mathrm{N})$} & \multicolumn{2}{c}{ Torque } \\
\hline Thumb & 0.03 & 0.12 & 0.61 & 2.16 \\
Index & 0.03 & 0.09 & 0.53 & 1.84 \\
Middle & 0.03 & 0.11 & 0.69 & 2.15 \\
Ring & 0.03 & 0.11 & 0.68 & 1.95 \\
Little & 0.04 & 0.11 & 0.82 & 1.80
\end{tabular}

TABLE 2: RMSE for each finger during calibration: with (left) and without (right) weight compensation (a) Experiment setup.

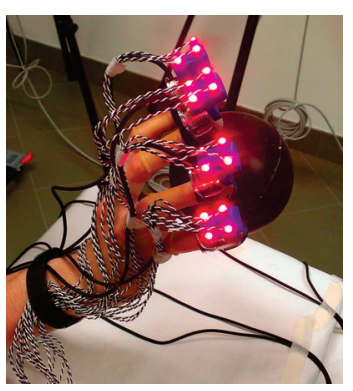

(b) Reconstruction and base reference frame $\{B\}$.

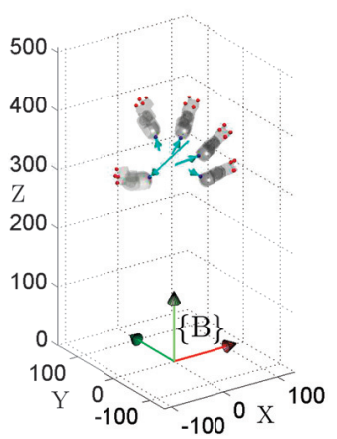

Fig. 9: Ball grasp and reconstruction.

\section{VALIDATION OF MEASUREMENTS}

In [20] a first validation of measurements from the device was performed, which however was mostly done in simple single-finger configurations. Complete force and contact reconstruction was obtained in a few sample experiments, but no quantitative validation was performed in these experiments. In this section we propose a validation of the device for full multifingered grasping, that uses classic robot grasping analysis in the framework presented by Bicchi in [6]. A subject (male, age 27) was asked to grasp two balls of different weights while wearing ThimbleSense on fingertips. The first object was a sponge ball (mass $m_{1}=0.053 \mathrm{Kg}$, radius $R_{1}=50 \mathrm{~mm}$ ) which was grasped with the whole hand (number of fingers used $n_{f}=5$ ), while the second was a tennis ball filled tightly with iron (mass $m_{2}=0.32$ $\mathrm{Kg}$, radius $R_{2}=33 \mathrm{~mm}$ ), which was grasped with four fingers $\left(n_{f}=4\right)$ owing to its smaller radius. In both cases the subject was instructed to lift the ball, squeeze it while holding it still with forces of different intensities, and put it down again. The validation procedure consisted of two different phases: in the first one the goal was to verify that the grasp equilibrium condition was fulfilled during the holding phase, while in the second we checked that the internal force variation was inside the null space of the grasp matrix. This way we are able to simultaneously validate all measurements of ThimbleSense.

Phase 1 required to verify that the vector of measured generalized forces $\mathbf{t} \in \mathbb{R}^{6 n_{f}}$, obtained from the fingertips measurements provided by ThimbleSenses, was correctly related to the applied external wrench $\mathbf{w} \in \mathbb{R}^{6}$ by the grasp equation

$$
\mathbf{w}=G \mathbf{t},
$$


(a) Force error components.

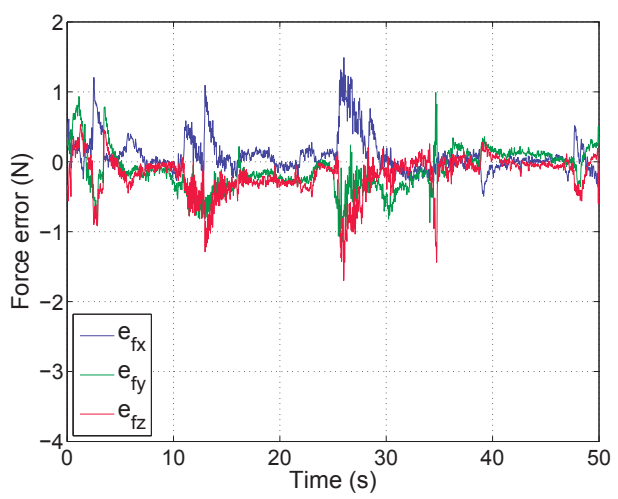

(b) Torque error components.

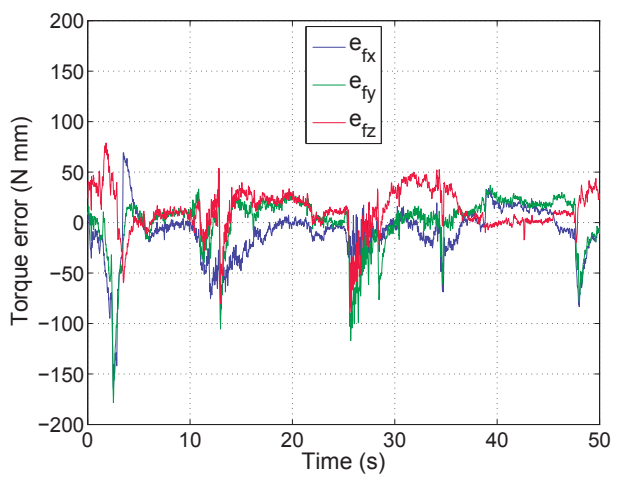

(c) Cartesian norm of forces on finger tips.

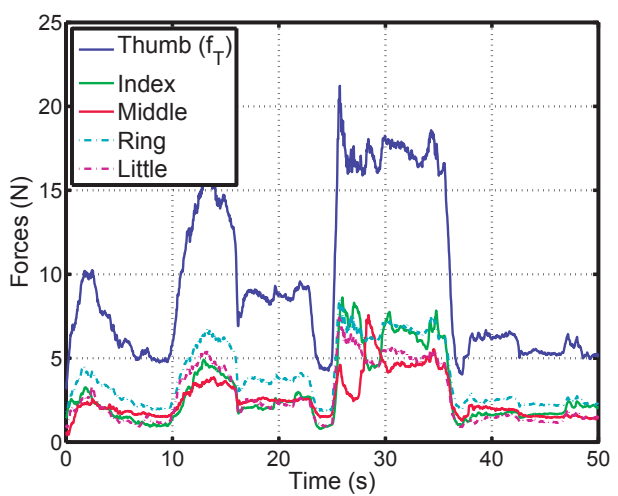

Fig. 10: Grasp analysis for the ball of mass $m_{1}=0.053 \mathrm{Kg}$.

which is valid under quasi-static conditions. The grasp matrix $G \in \mathbb{R}^{6 \times 6 n_{f}}$ can be written as

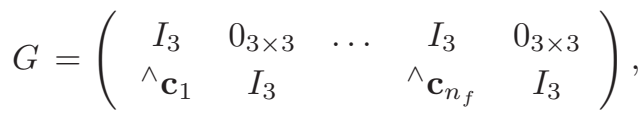

where $^{\wedge}(\cdot)$ is the skew-matrix operator and $\mathbf{c}_{1} \ldots \mathbf{c}_{n_{f}}$ are the contact points coordinates calculated through the intrinsic tactile sensing algorithm, expressed in a reference system with origin in the center of mass of the ball $\mathbf{b}$ and oriented as the fixed base frame $\{B\}$.

Since the ball mass $m$ is known, we can assign a nominal value for the external wrench $\mathbf{w}=\overline{\mathbf{w}}=[0,0,-m g, 0,0,0]^{T}$, with $g=9.81 \mathrm{~m} / \mathrm{s}^{2}$. The residual error $\mathbf{e}$ is thus computed as

$$
\mathbf{e}:=\overline{\mathbf{w}}-G \mathbf{t}=\left[e_{f_{x}} e_{f_{y}} e_{f_{z}} e_{\tau_{x}} e_{\tau_{y}} e_{\tau_{z}}\right]^{T},
$$

(a) Force error components.

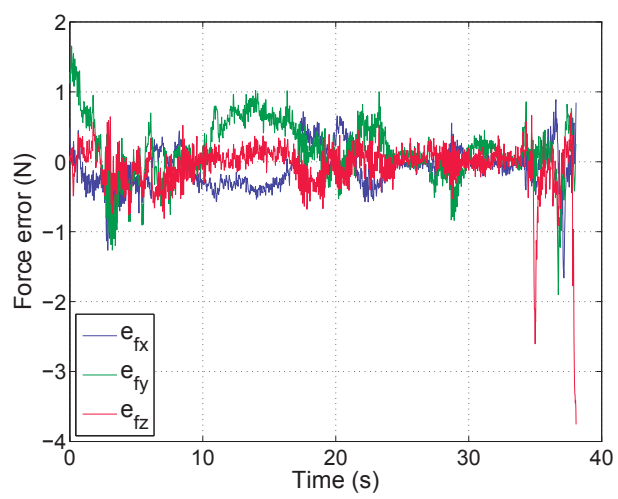

(b) Torque error components.

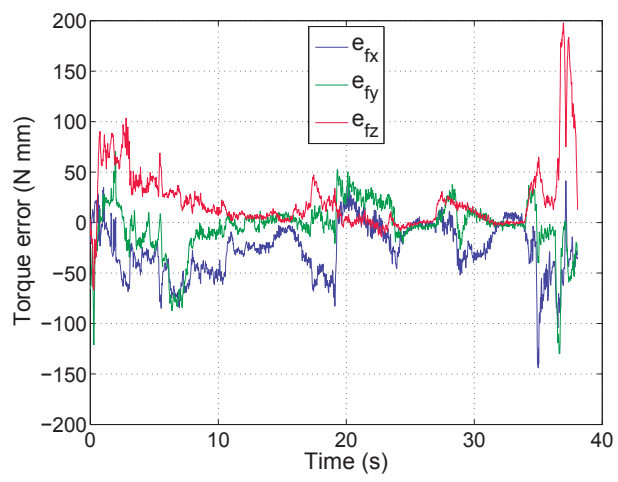

(c) Cartesian norm of forces on finger tips.

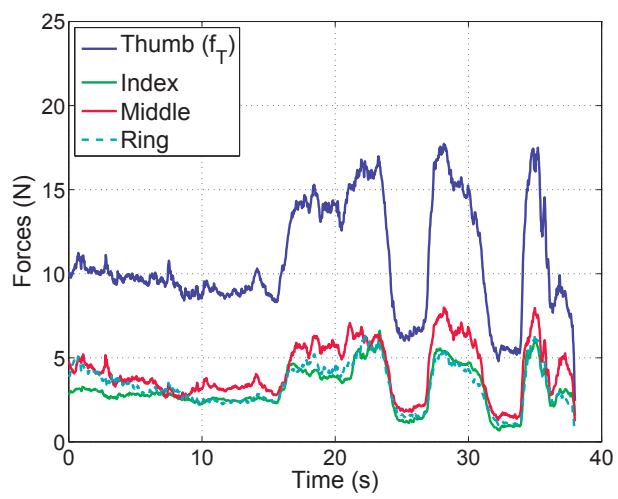

Fig. 11: Grasp analysis for the ball of mass $m_{2}=0.32 \mathrm{Kg}$.

where $\left[\begin{array}{lll}e_{f_{x}} & e_{f_{y}} & e_{f_{z}}\end{array}\right]$ and $\left[\begin{array}{lll}e_{\tau_{x}} & e_{\tau_{y}} & e_{\tau_{z}}\end{array}\right]$ are the force and torque component error vectors, respectively. Figures 10 and 11 show plots of the result for two trials. The following observations can be made:

- The overall grasp error tends to increase when fingertip forces are higher;

- There is a transition at the beginning and at the end of the task where errors are relatively higher even if grasp forces are low: this is caused by the fact that the model we are using to calculate error is quasi-static, and as such does not take into account dynamic actions (equation (1) no longer holds true when the ball is moving).

Therefore, it can be interesting to consider a force per- 
(a) Ball position $\mathbf{b}$ in space.

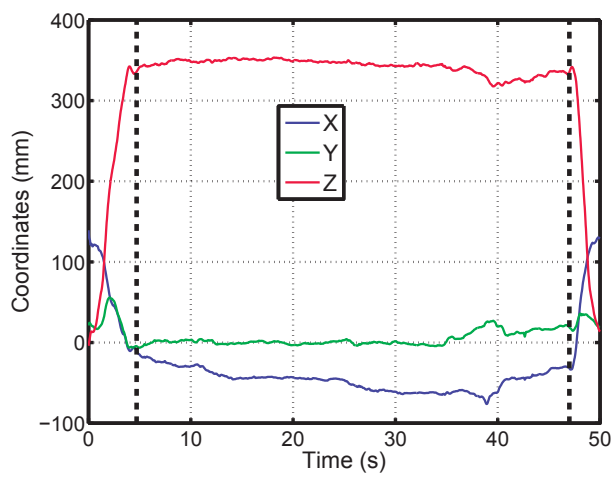

(b) Normalized force error.

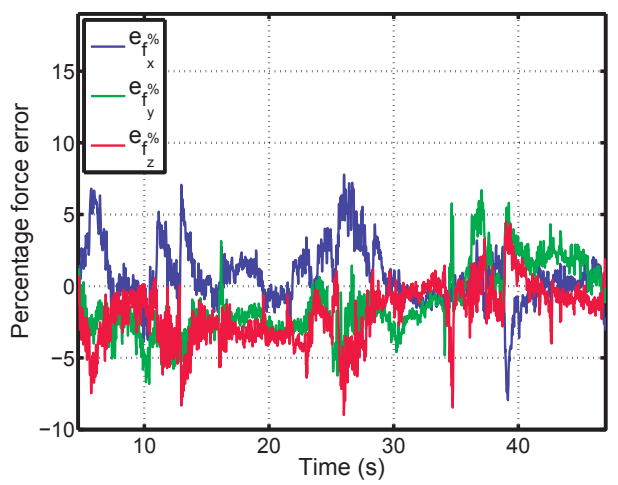

Fig. 12: Error normalization for the ball of mass $m_{1}=0.053$ $\mathrm{Kg}$.

centage error, defined as

$$
\mathbf{e}_{f \%}:=\left[e_{f_{x}^{\%}} e_{f_{y}^{\%}} e_{f_{z}^{\%}}\right]=100\left[\frac{e_{f_{x}}}{f_{T}} \frac{e_{f_{y}}}{f_{T}} \frac{e_{f_{z}}}{f_{T}}\right]^{T},
$$

where $f_{T}$ is the norm of the force applied by the thumb on the object, which was chosen as indication of the intensity of grasp. The percentage error is considered for the time frame during which the ball is held still. In order to identify such time frame, first we estimated the position of the center of mass of the ball over time. For each finger, an estimate $\mathbf{b}_{i}$ of the ball center can be obtained as

$$
\mathbf{b}_{i}=\mathbf{c}_{i}+R \mathbf{n}_{i}
$$

where $R$ is the radius of the ball in $\mathrm{mm}$ and $\mathbf{n}_{i}$ is the unit vector normal to the contact surface during grasp, which can be obtained from the intrinsic tactile algorithm. The global ball center position estimate $\mathbf{b}$ can then be obtained as

$$
\mathbf{b}=\frac{1}{n_{f}} \sum_{i=1}^{n_{f}} \mathbf{b}_{i} \text {. }
$$

This can be used to distinguish the initial and final transient phases from the holding phase. Figures 12a-13a show plots of $\mathbf{b}$ for the two trials considered, each with the time frame of interest highlighted by two vertical dashed lines, while Figures $12 \mathrm{~b}-13 \mathrm{~b}$ show plots of the percentage error during such time frame. Tables 3 and 4 report numerical values of both absolute and percentage force Root Mean Square Error (RMSE) for both tasks. Torque error is also reported. (a) Ball position $\mathbf{b}$ in space.

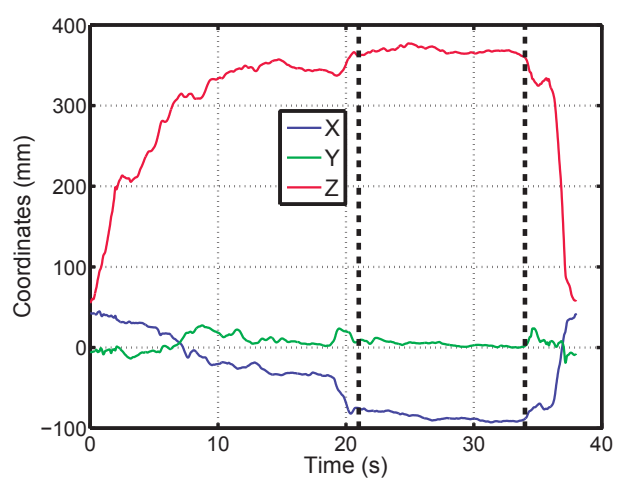

(b) Normalized force error.

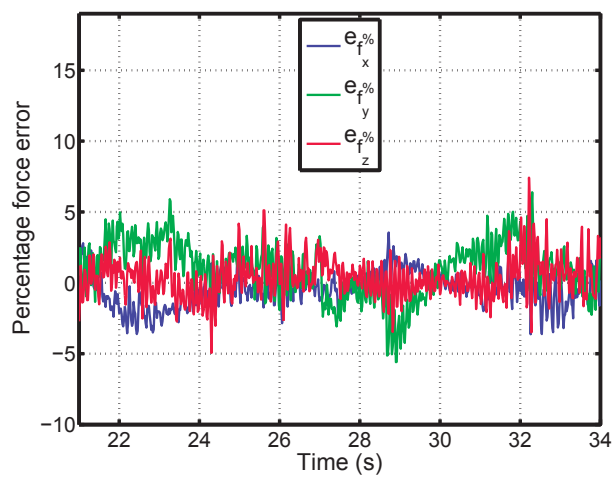

Fig. 13: Error normalization for the ball of mass $m_{2}=0.32$ $\mathrm{Kg}$.

\begin{tabular}{cccc}
\hline Components & $f_{x}$ & $f_{y}$ & $f_{z}$ \\
\hline RMSE & $0.27[\mathrm{~N}]$ & $0.30[\mathrm{~N}]$ & $0.34[\mathrm{~N}]$ \\
Percentage RMSE & $2.22 \%$ & $2.66 \%$ & $2.79 \%$
\end{tabular}

\begin{tabular}{cccc}
\hline Components & $\tau_{x}[\mathrm{Nmm}]$ & $\tau_{y}[\mathrm{Nmm}]$ & $\tau_{z}[\mathrm{Nmm}]$ \\
\hline RMSE & 19.80 & 22.11 & 18.52
\end{tabular}

TABLE 3: Error for experiments with the ball of mass $m_{1}=$ $0.053 \mathrm{Kg}$.

It is worth noting that there is no intuitive choice of a normalization quantity for torques; for this reason only absolute torque error is shown.

Phase 2 of the validation exploits the force variation during the task. Let us consider $\Delta \mathbf{t}=\mathbf{t}_{k}-\mathbf{t}_{0}$, where $\mathbf{t}_{0}$ is the generalized force measured for an initial sample $s_{0}$ and $\mathbf{t}_{k}$ is the force measured in a later sample $s_{k}$. Since the ball is held still, the external wrench $\mathbf{w}$ is not changing, and from $\mathbf{w}=G \mathbf{t}_{0}=G \mathbf{t}_{k} \forall k$ follows that $\Delta \mathbf{t}$ lies in the

\begin{tabular}{cccc}
\hline Components & $f_{x}$ & $f_{y}$ & $f_{z}$ \\
\hline RMSE & $0.17[\mathrm{~N}]$ & $0.29[\mathrm{~N}]$ & $0.15[\mathrm{~N}]$ \\
Percentage RMSE & $1.28 \%$ & $2.17 \%$ & $1.46 \%$ \\
& & & \\
\hline Components & $\tau_{x}[\mathrm{Nmm}]$ & $\tau_{y}[\mathrm{Nmm}]$ & $\tau_{z}[\mathrm{Nmm}]$ \\
\hline RMSE & 13.68 & 16.18 & 8.44
\end{tabular}

TABLE 4: Error for experiments with the ball of mass $m_{2}=$ $0.32 \mathrm{Kg}$. 


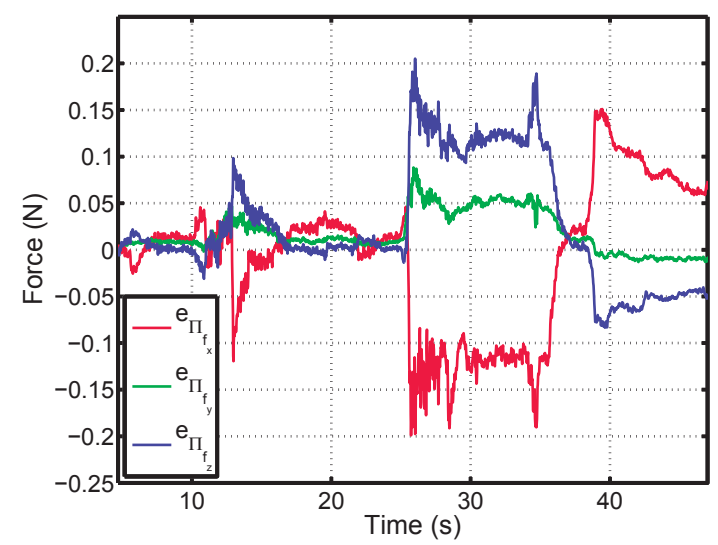

Fig. 14: Internal force projection error components for the task with $n_{f}=5$.

\begin{tabular}{cccccc}
\hline Components & Thumb & Index & Middle & Ring & Little \\
\hline \multicolumn{7}{c}{$m_{1}=0.053 \mathrm{Kg}$} \\
\hline$f_{x}[\mathrm{~N}]$ & 0.077 & 0.10 & 0.085 & 0.082 & 0.073 \\
$f_{y}[\mathrm{~N}]$ & 0.036 & 0.72 & 0.17 & 0.034 & 0.10 \\
$f_{z}[\mathrm{~N}]$ & 0.075 & 0.068 & 0.14 & 0.15 & 0.32 \\
\hline \multicolumn{7}{c}{$m_{2}=0.32 \mathrm{Kg}$} \\
\hline$f_{x}[\mathrm{~N}]$ & 0.143 & 0.41 & 0.34 & 0.14 & $\mathrm{n} / \mathrm{a}$ \\
$f_{y}[\mathrm{~N}]$ & 0.164 & 0.074 & 0.20 & 0.026 & $\mathrm{n} / \mathrm{a}$ \\
$f_{z}[\mathrm{~N}]$ & 0.0063 & 0.054 & 0.030 & 0.13 & $\mathrm{n} / \mathrm{a}$
\end{tabular}

TABLE 5: RMSE for the force components of $\mathbf{e}_{\Pi}$.

nullspace of $G(\Delta \mathbf{t} \in \mathcal{N}(G))$. It is known from grasp theory and linear algebra ([6], Meyer [28]) that $\left(I-G^{+} G\right)$, where $I$ is the identity matrix, is a projector to $\mathcal{N}(G)$. Therefore, if we compute

$$
\Pi_{G}(\Delta \mathbf{t}):=\left(I-G^{+} G\right) \Delta \mathbf{t}=\left[I-G^{T}\left(G G^{T}\right)^{-1} G\right] \Delta \mathbf{t}
$$

since $\Delta \mathbf{t} \in \mathcal{N}(G)$ it should be true that $\Pi_{G}(\Delta \mathbf{t})=\Delta \mathbf{t}$. We can then define the error

$$
\begin{aligned}
\mathbf{e}_{\Pi} & :=\Pi_{G}(\Delta \mathbf{t})-\Delta \mathbf{t}= \\
& =\left[e_{\Pi_{f x}} e_{\Pi_{f_{y}}} e_{\Pi_{f_{z}}} e_{\Pi_{\tau_{x}}} e_{\Pi_{\tau_{y}}} e_{\Pi_{\tau_{z}}}\right]^{T} .
\end{aligned}
$$

As an example, figure 14 shows the force components of such error for the trial with the ball of mass $m_{1}$. A more complete representation of results can be found in Table 5, which shows RMSE considering only force components for the sake of space. RMSE for torques were all of the order of $10^{-3} \mathrm{~N} \mathrm{~mm}$.

\section{TACTILE FEEDBACK IMPAIRMENT EVALUATION}

The experiment shown in the previous section focused on validating the ThimbleSense measurements. However, there is another important aspect that needs to be analyzed. When human users wear the ThimbleSense, a rigid shell is placed on fingertips, which inevitably alters cutaneous perception and hence force modulation. In particular we expected subjects to exert larger forces to compensate for the distortion in tactile feedback, whose importance in grip control is well known as we discussed in the Introduction. However,

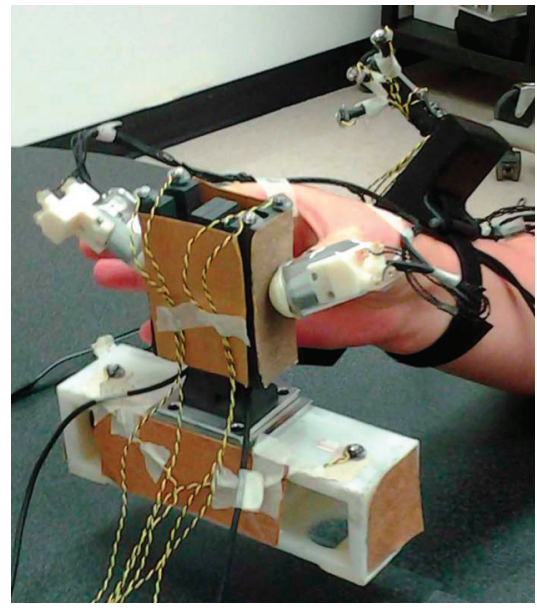

Fig. 15: Inverted T experiment setup

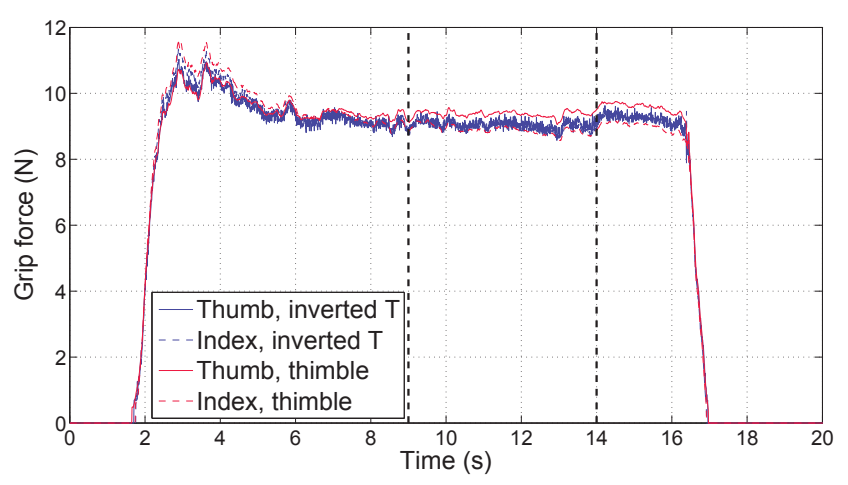

Fig. 16: Grip force data for a representative trial.

as subjects familiarize themselves with the ThimbleSense and the task, we also expected forces to decrease. In this section we describe an example of a typical experiment in neuroscientific studies on human grasp control, which is performed first while wearing ThimbleSense and then with bare fingers. The aim is studying the evolution of grasp forces under the two conditions, with the two-fold goal of evaluating the effect of cutaneous distortion and possibility of reducing it through practice.

We employed the grip device with the inverted $\mathrm{T}$ design used by Zhang et al. in [14]. A total of 4 LED markers were added on the top to allow estimation of position and orientation of the sensorized object. The total mass of the object was $730 \mathrm{~g}$, with the center of mass of the system located in the middle plane. Figure 15 shows the experimental apparatus. The task consisted in lifting the object using thumb and index of the right hand. We asked 8 subjects ( 7 males and 1 female, age $28.2 \pm 2.8,5$ right handed and 3 left handed) to perform the experiment, in two different sessions. All subjects were naive to the use of ThimbleSense, all had no previous history of orthopedic or neurological pathology or trauma to the upper limbs, and all gave their informed consent. The protocols were approved by the Arizona State University Office of Research Integrity and Assurance. During the first session subjects wore the ThimbleSense on the right hand thumb and index fingertips, while for the second session they were asked to perform 
the same task with bare fingertips. Both sessions consisted in 30 trials each and at least two days were interposed between one session and the other. Subjects could take breaks whenever they needed, and were given a minimum of a one-minute break after the 15th trial, in order to prevent fatigue.

It is known from literature (e.g., [7]) that grip force during manipulation is regulated with a relatively low margin with respect to the slip condition. Thus, we can use the difference in the mean steady-state grip force under the two conditions as an indication of how much the grasp is being distorted by the thimbles. Note, however, that large differences between the static friction coefficients (bare fingertips versus latex artificial finger pad against the sandpaper covering the graspable surface of the object) might also have contributed to elicit different grip forces. To further investigate this aspect we asked a male righthanded subject (27 year old), with no history of neurological or physical impairment, and who did not participate to the main experiment, to perform 5 trials of a standard slip test for both conditions in order to get an approximate estimation of the static friction coefficient, following a procedure similar to the one used by Baud-Bovy et al. in [18]. The average value of the static friction coefficient obtained for the bare fingertip condition (1.20) was similar to the one reported in [18], while the average value obtained with the ThimbleSense devices was 1.17. This result led us to neglect the difference in the friction coefficient between the two experimental conditions for subsequent force analyses.

Figure 16 shows the grip force from a representative trial. Both steady-state mean grip during the hold phase and grip peak during lift were considered. For what concerns the first, since we were interested in steady-state values, we considered a time window of 5 seconds for each trial, during which the contact between fingertips and the object was stable (grip force stardard deviation less than $1.5 \mathrm{~N}$ ). The mean grip value for each trial, subject, and experimental condition was computed as the average of measurements in this time window (Figures 17-20). As we expected, the average grip force started higher when wearing the thimbles compared to the bare fingertip condition. However, it can be observed that the mean grip forces exerted when wearing the ThimbleSense also tended to decrease across trials, which for some subjects yielded a final value that was comparable to the one obtained when performing the task with bare fingertips (Figure 18 and Figure 20), whereas for other subjects the value remained higher. Since the cutaneous afferents distorted by the thimbles play a major role during the dynamic phase of grasp [8], we considered also grip peak during the lifting phase, which is also shown in Figures 17-20. It can be seen that the behavior is similar to what observed for the steady-state mean grip.

We attempted to quantify the grip forces adaptation rate in two different ways. The first approach was a linear interpolation across trials. This analysis provides the slope of the linear model for forces $F$ as function of number of trials $n_{t}$, $F=\alpha n_{t}+\beta$, as a numerical indication of adaptation rate. Tab. 6 shows the numerical results of this analysis applied on steady-state mean grip during hold and grip peak during lift, for all subjects and for both experimental conditions. It can be seen that the slope $\alpha$ is negative and always higher
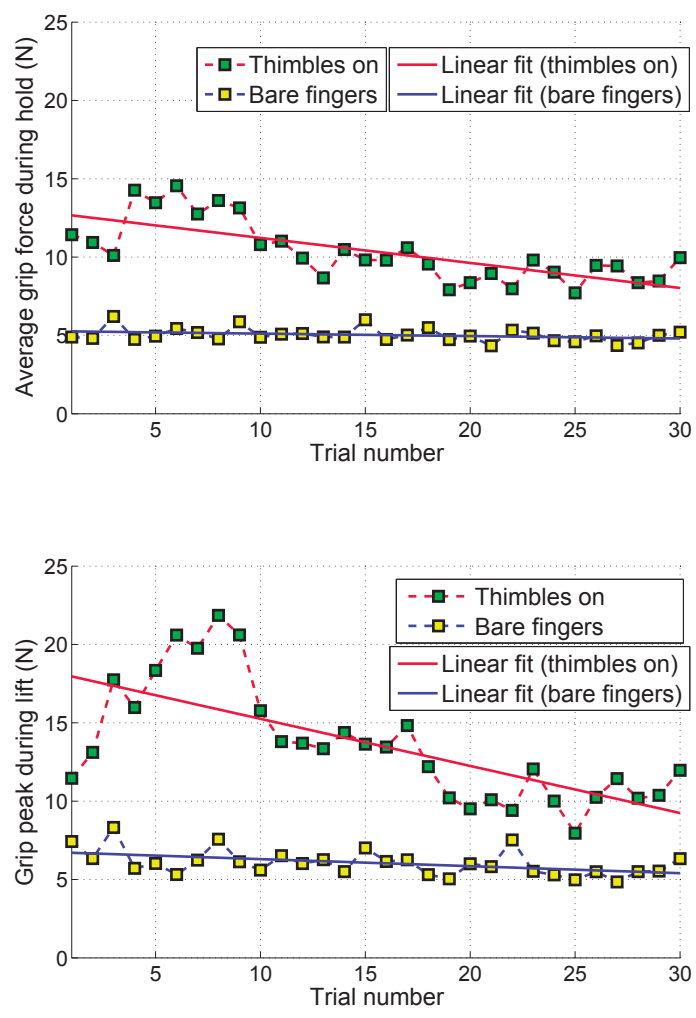

Fig. 17: Subject 3 (right-handed).
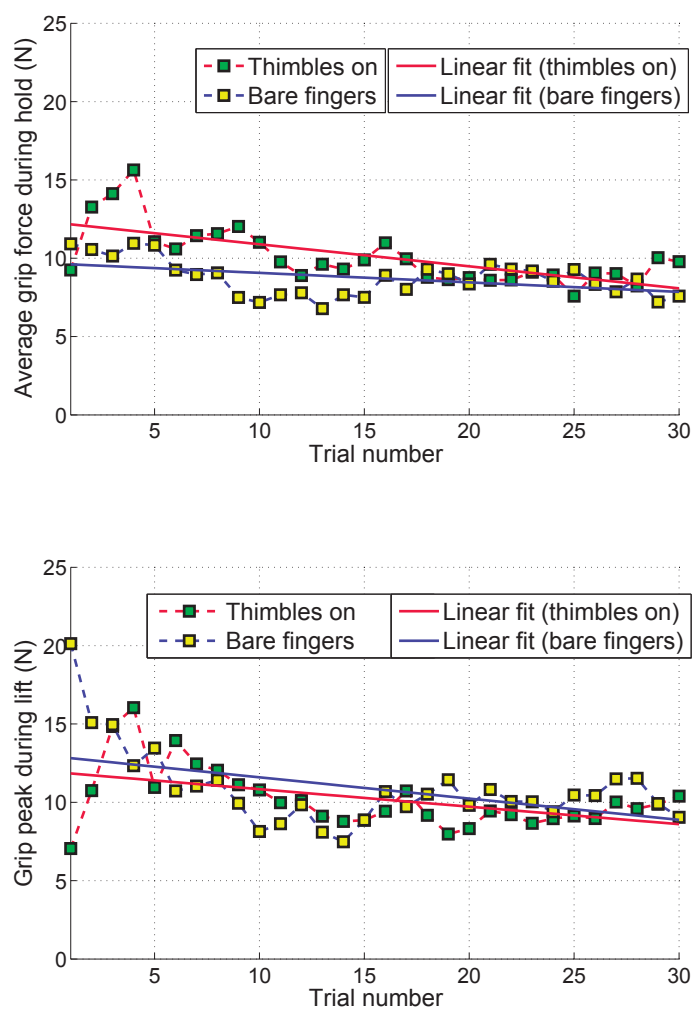

Fig. 18: Subject 5 (right-handed). 

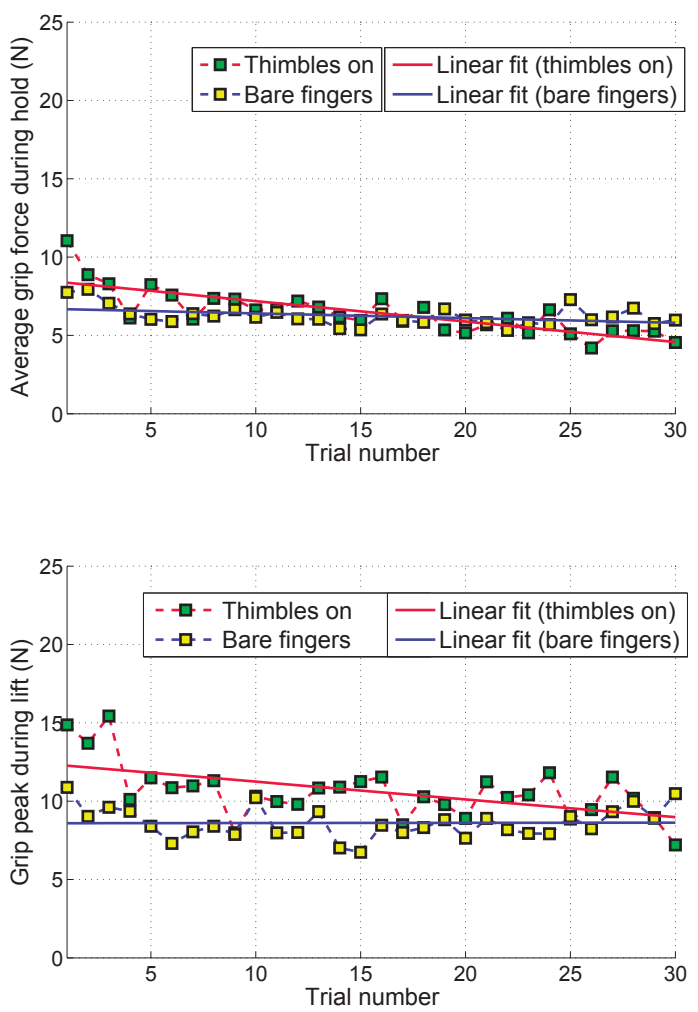

Fig. 19: Subject 6 (left-handed).
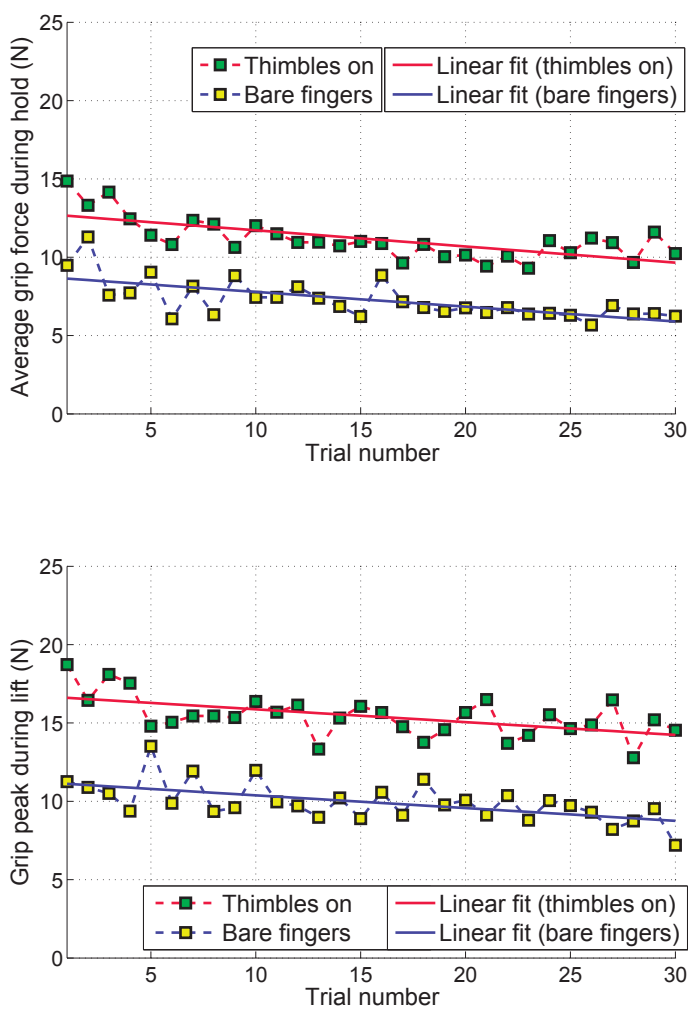

Fig. 20: Subject 7 (left-handed).

\begin{tabular}{|c|c|c|c|c|c|}
\hline \multirow{2}{*}{ Subject } & \multicolumn{2}{|c|}{ Average grip during hold } & \multicolumn{2}{|c|}{ Grip peak during lift } & \\
\hline & $\mathrm{TH}$ & $\mathrm{BF}$ & $\mathrm{TH}$ & $\mathrm{BF}$ & \\
\hline \multirow{2}{*}{1 (R) } & -0.107 & -0.0553 & -0.141 & -0.130 & $\alpha$ \\
\hline & $1.17 \mathrm{e}-06$ & 0.0113 & $3.58 \mathrm{e}-05$ & $1.82 \mathrm{e}-04$ & $\mathrm{p}$ value \\
\hline \multirow{2}{*}{2 (R) } & -0.0598 & -0.0290 & -0.136 & -0.129 & $\alpha$ \\
\hline & 0.0293 & 0.0761 & $1.64 \mathrm{e}-04$ & $1.69 \mathrm{e}-03$ & $p$ value \\
\hline \multirow{2}{*}{$3(\mathrm{R})$} & -0.131 & -0.0160 & -0.301 & -0.0450 & $\alpha$ \\
\hline & $4.85 \mathrm{e}-08$ & 0.191 & $1.38 \mathrm{e}-05$ & $7.82 \mathrm{e}-03$ & $\mathrm{p}$ value \\
\hline \multirow{2}{*}{$4(\mathrm{R})$} & -0.131 & 0.0299 & -0.184 & -0.0112 & $\alpha$ \\
\hline & 7.33e-08 & 0.0218 & $1.3 \mathrm{e}-07$ & 0.4153 & $p$ value \\
\hline \multirow{2}{*}{$5(\mathrm{R})$} & -0.103 & -0.0943 & -0.111 & -0.136 & $\alpha$ \\
\hline & $2-02 \mathrm{e}-05$ & $4.84 \mathrm{e}-05$ & $5.38 \mathrm{e}-03$ & 7.13e-03 & $\mathrm{p}$ value \\
\hline \multirow{2}{*}{$6(\mathrm{~L})$} & -0.160 & -0.0145 & -0.113 & 0.0012 & $\alpha$ \\
\hline & $5.70 \mathrm{e}-06$ & 0.128 & $1.31 \mathrm{e}-03$ & 0.955 & $p$ value \\
\hline \multirow{2}{*}{$7(\mathrm{~L})$} & -0.141 & -0.0606 & -0.0817 & -0.0815 & $\alpha$ \\
\hline & 0.388 & 0.612 & $1.71 \mathrm{e}-03$ & $8.49 \mathrm{e}-04$ & $\mathrm{p}$ value \\
\hline \multirow{2}{*}{$8(\mathrm{~L})$} & -0.286 & -0.0757 & -0.150 & -0.0907 & $\alpha$ \\
\hline & 0.388 & 0.612 & $1.54 \mathrm{e}-04$ & $8.66 \mathrm{e}-03$ & $p$ value \\
\hline
\end{tabular}

TABLE 6: Mean grip linear fit slope $\alpha$ and $\mathrm{p}$ value. (R) indicates right-handed subjects, while (L) indicates lefthanded subjects; TH indicates ThimbleSense being placed on the fingers, while BF indicates the bare fingers condition.

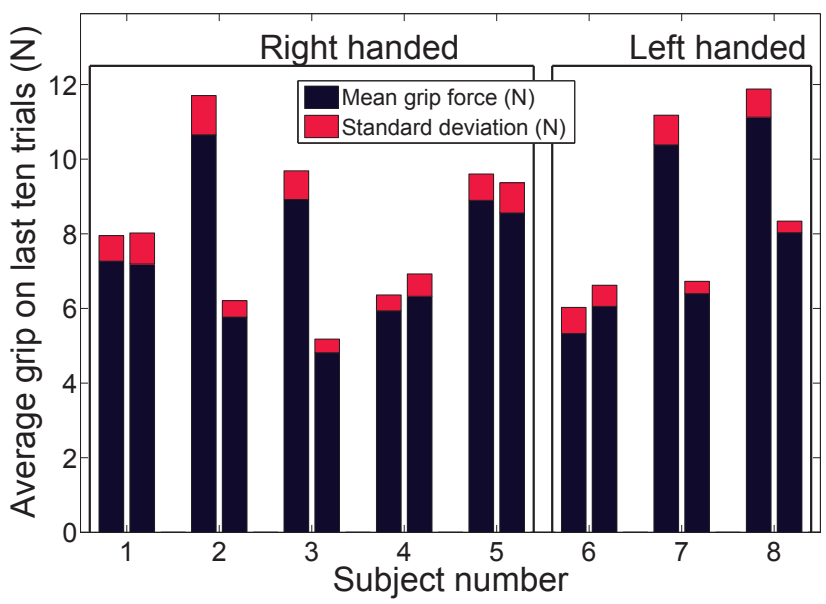

Fig. 21: Average of the mean grip on the last ten trials for each subject. There are two bars for each subject: the first bar represents values while wearing ThimbleSense, while the second bar is for bare fingers.

when subjects wear the ThimbleSense, and p-values show that the value of this slope is always statistically significant, except for some subjects under the bare fingertip condition who had grip forces which immediately approached steady state values. The negative and larger slopes of the linear fit to grip forces across trials indicate that grip forces tended to decrease with practice, and at a higher rate than when using bare fingertips. It is worth pointing out that the linear fit is not meant as an actual model of the process, but it is used as an indication of whether there is learning or not.

The second approach we used to quantify adaptation was to compare grip force over the latest trials between the two experimental conditions. Figure 21 shows the comparison of the average of the steady-state mean grip during hold 


\begin{tabular}{|c|c|c|c|}
\hline \multirow{2}{*}{ Subject } & \multicolumn{2}{|c|}{ Mean grip force $(\mathrm{N})$} & \multirow{2}{*}{$\mathrm{p}$-value } \\
\hline & TH & $\mathrm{BF}$ & \\
\hline $1(\mathrm{R}) \dagger$ & $7.26 \pm 0.69$ & $7.19 \pm 0.83$ & 0.920 \\
\hline $4(\mathrm{R}) *$ & $5.94 \pm 0.43$ & $6.33 \pm 0.60$ & 0.0506 \\
\hline $5(\mathrm{R}) *$ & $8.9 \pm 0.71$ & $8.56 \pm 0.81$ & 0.471 \\
\hline $6(\mathrm{~L}) *$ & $5.33 \pm 0.70$ & $6.06 \pm 0.57$ & 0.0546 \\
\hline
\end{tabular}

TABLE 7: Steady state mean grip. Paired t-test was used for data marked with $*$, while Wilcoxon signed rank test was used for data marked with $\dagger$. Normality was verified with Lilliefors test.

(a) Steady-state mean grip during hold for subject 2 .

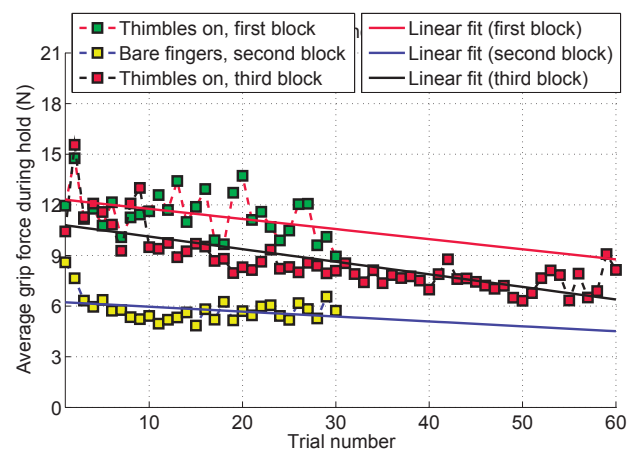

(b) Grip peak during lift for subject 2.

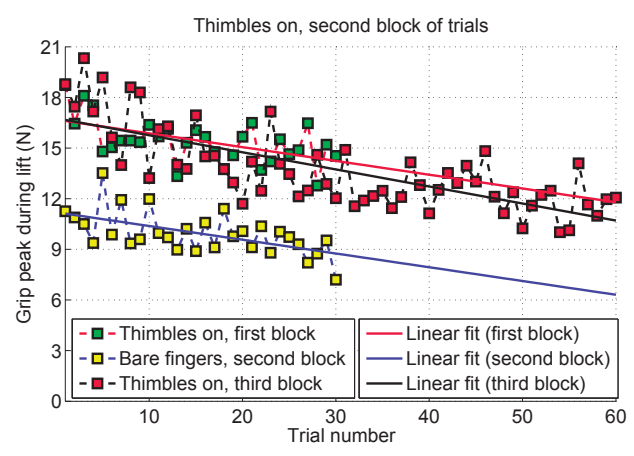

Fig. 22: Comparison between the three blocks of trials performed on subject 2 .

over the last ten trials from the two conditions and each subject (note that subjects 1 to 5 are right-handed, while subjects 6 to 8 are left-handed). As it was already observed when comparing the sample plots of mean grip over number of trials, results varied across subjects. Specifically, subjects number 1, 4, 5 and 6 exhibited comparable values for the mean grip over the last ten trials in the two conditions, for which statistical analysis revealed no statistically significant difference (Table 7). The other half of the subjects, however,

\begin{tabular}{lcccc}
\hline \multirow{2}{*}{ Subject } & Average grip during hold & & Grip peak during lift & \\
\cline { 2 - 2 } & $\mathrm{TH}(30$ trials $)$ & & $\mathrm{TH}(60$ trials $)$ & \\
\hline \multirow{2}{*}{$2(\mathrm{R})$} & -0.074 & & -0.101 & $\alpha$ \\
& $1.64 \mathrm{e}-12$ & & $1.08 \mathrm{e}-11$ & $\mathrm{p}$-value \\
\hline
\end{tabular}

TABLE 8: Linear fit goodness for the 60-trials block. still exhibited a significantly higher grip force when wearing the ThimbleSense.

These results indicate that at least half of the subjects were able to adapt their steady-state grip forces, despite the distortion of tactile sensation, to a level comparable to the one found when using bare fingertips. To further investigate this phenomenon, a third block of trials was presented to subject 2 who exhibited the largest difference between the final values of grip force in the two experimental conditions. For this third block of trials, the subject was asked to repeat the task while wearing the thimbles, but the number of trials was increased from 30 to 60 to provide him with more time to become familiar with ThimbleSense and the task. The third block of trials was performed 11 days after the subjects had performed the second block of trials.

Figure 22 shows the grip force behavior for subject 2 for all three blocks of trials: the average of steady-state mean grip during hold on the last ten trials was $10.65 \pm 1.05$, $5.77 \pm 0.44$ and $7.53 \pm 0.87 \mathrm{~N}$ for each block respectively. The final average value of force, while still higher than the final value of grip force in the bare fingertip condition, was considerably smaller than the value that was obtained in the first block. Most importantly, the comparison of data from the first and third blocks of trials reveals that the grip force decreased more rapidly during the third block (this can be quantified numerically by comparing values of $\alpha$ in Tab. 6 and 8). The initial force $\beta$ was also smaller. Therefore, these data indicate that the subject who initially exhibited the weakest force adaptation benefited from longer practice with ThimbleSense and the task ( $\alpha$ coefficient significantly smaller than zero), as well as from the previous practice (smaller initial force and more rapid decrease of grip force relative to the first block, which was carried in the same condition as the third).

From these results, we can not claim that a complete compensation of cutaneous feedback distortion can be obtained with training. However, this experiment confirms that there is some learning, be it device or task related. For this reason we believe that ThimbleSense could be profitably used in experiments on anticipatory control (e.g. [16]) with distortion of cutaneous feedback. Targeted studies can be focused on the kinaesthetic aspect of grasp control with applications on design and sensing of artificial hands and prostheses, as we further develop in the Conclusions. Finally, it is worth noting that the object never slipped and was never dropped during the experiments. This, together with the fact that the same object was always used with no shape or size change, minimized the role of visual cues in the experiment.

\section{Conclusions}

In this work, we described the ThimbleSense, a wearable device that allows us to obtain measurements of forces applied during grasping as well as to estimate the position of the contact points. Experimental validation evaluated the accuracy of measurements on grasps of two different balls, leading in both cases to force RMSE less than $0.35 \mathrm{~N}$ (less than $3 \%$ of thumb force norm) and torque RMSE less than 23 $\mathrm{N} \mathrm{mm}$. We also performed a comparison of grip force evolution for a simple thumb-index lifting task, performed with 
(a) Fingertip detail.

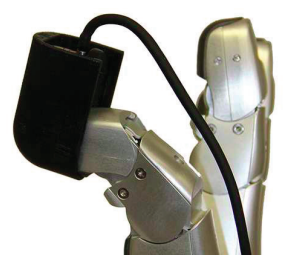

(b) Object grasp.

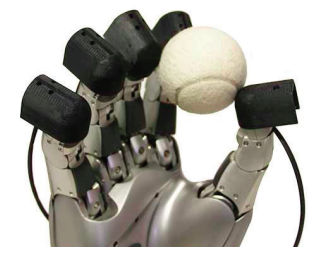

Fig. 23: Example of application of ThimbleSense on a robot (DLR Hand II).

and without thimbles. This comparison showed a reduction of grip force over the number of trials: indeed, for half of the subjects the same value of mean steady state grip force was measured for both conditions. Owing to the fact that only a single simple task was considered, we can not conclude from this that there was a learning specifically related to the device: however, we can observe that learning during the experiment was not completely hampered by wearing ThimbleSense, as instead would happen for subjects who completely lost all somatosensory feedback ([7], [9]).

Thanks to its versatility in providing complete measurements of forces and contact points during multifingered grasps of variously shaped objects, ThimbleSense shows promise to be a powerful tool in the field of wearable haptics and human hand behavior studies, as long as the limitations related to the cutaneous cues distortion are kept in due consideration. Future work will focus on studies on reactive grasp and force synergies under impaired cutaneous feedback, to be used to improve the design of robotic hands and prostheses, e.g. to implement low-level grasp reflexes and for studies on grasp stability. Indeed, the device can be adapted with minor modifications to robotic manipulators (Figure 23 shows a preliminary application on the DLR Hand II), to provide a complete set of force and contact point measurements to study grasp stability.

\section{ACKNOWLEDGMENTS}

The authors gratefully acknowledge Qiushi Fu, Manolo Garabini, Pranav J. Parikh, Patrick McGurrin, Keivan Mojtahedi, and Daisuke Shibata for their valuable advices, and Andrea Di Basco for his unique contribution in the physical realization of the device. This work was partially supported by the European Community funded projects WEARHAP, PACMAN and SOMA (contracts 601165, 600918 and 645599 respectively), by the ERC Advanced Grant no. 291166 SoftHands and by the Collaborative Research Grant BCS-1153034 to M. Santello from the National Science Foundation (NSF).

\section{RefEREnCES}

[1] J. R. Flanagan, M. C. Bowman, and R. S. Johansson, "Control strategies in object manipulation tasks," Current Opinion in Neurobiology, vol. 16, no. 6, pp. 650-659, 2006.

[2] R. S. Johansson and J. R. Flanagan, "Coding and use of tactile signals from the fingertips in object manipulation tasks," Nature Reviews Neuroscience, vol. 10, no. 5, pp. 345-359, 2009.

[3] D. M. Wolpert, J. Dierichsen, and J. R. Flanagan, "Principles of sensorimotor learning," Nature Reviews Neuroscience, vol. 12, pp. 739-751, 2011.
[4] M. R. Cutkosky and R. D. Howe, "Human grasp choice and robotic grasp analysis," Dextrous robot hands, pp. 5-31, 1990.

[5] R. S. Johansson and I. Birznieks, "First spikes in ensembles of human tactile afferents code complex spatial fingertip events," Nature neuroscience, vol. 7, no. 2, pp. 170-177, 2004.

[6] A. Bicchi, "On the problem of decomposing grasp and manipulation forces in multiple whole limb manipulation," Int. Journal of Robotics and Autonomous Systems, vol. 13, no. 2, pp. 127 - 147, 1994.

[7] R. S. Johansson and G. Westling, "Roles of glabrous skin receptors and sensorimotor memory in automatic control of precision grip when lifting rougher or more slippery objects," Experimental brain research, vol. 56, no. 3, pp. 550-564, 1984.

[8] G. Westling and R. S. Johansson, "Responses in glabrous skin mechanoreceptors during precision grip in humans," Experimental Brain Research, vol. 66, no. 1, pp. 128-140, 1987.

[9] D. A. Nowak, S. Glasauer, and J. Hermsdörfer, "How predictive is grip force control in the complete absence of somatosensory feedback?" Brain, vol. 127, no. 1, pp. 182-192, 2004.

[10] L. Dipietro, A. M. Sabatini, and P. Dario, "A survey of glove-based systems and their applications," IEEE Transactions on systems, man and cybernetics, vol. 38, no. 4, pp. $461-482,2008$.

[11] T. R. Grieve, J. M. Hollerbach, and S. A. Mascaro, "Force prediction by fingernail imaging using active appearance models," in Proceedings of IEEE World Haptics Conference, 2013.

[12] M. Santello and J. F. Soechting, "Force synergies for multifingered grasping," Experimental Brain Research, vol. 133, no. 4, pp. 457 $467,2000$.

[13] S. A. Winges, S. E. Eonta, J. F. Soechting, and M. Flanders, "Multidigit control of contact forces during rotation of a hand-held object," Journal of neurophysiology, vol. 99, no. 4, pp. 1846-1856, 2008.

[14] W. Zhang, A. M. Gordon, Q. Fu, and M. Santello, "Manipulation after object rotation reveals independent sensorimotor memory representations of digit positions and forces," The Journal of Neurophysiology, vol. 103, no. 6, pp. 2953 - 2964, 2010.

[15] V. M. Zatsiorsky and M. L. Latash, "Multi-finger prehension: An overview," Journal of Motor Behavior, vol. 40, no. 5, pp. 446-476, 2008.

[16] Q. Fu, W. Zhang, and M. Santello, “Anticipatory planning and control of grasp positions and forces for dexterous two-digit manipulation," The Journal of Neuroscience, vol. 30, no. 27, pp. 9117 - 9126, 2010.

[17] Q. Fu, Z. Hasan, and M. Santello, "Transfer of learned manipulation following changes in degrees of freedom," The Journal of Neuroscience, vol. 31, no. 38, pp. 13576 - 13584, 2011.

[18] G. Baud-Bovy and J. F. Soechting, "Two virtual fingers in the control of the tripod grasp," Journal of Neurophysiology, vol. 86, no. 2, pp. 604-615, 2001.

[19] S. A. Winges, S. E. Eonta, J. F. Soechting, and M. Flanders, "Effects of object compliance on three-digit grasping," Journal of Neruphysiology, vol. 101, no. 5, pp. 2447-2458, 2009.

[20] E. Battaglia, G. Grioli, M. G. Catalano, M. Santello, and A. Bicchi, "Thimblesense: an individual-digit wearable tactile sensor for experimental grasp studies," in Proceedings of IEEE International Conference on Robotics and Automation, 2014.

[21] C. G. Atkeson, C. H. An, and J. M. Hollerbach, "Rigid body load identification for manipulators," in Proceedings of IEEE Conference on Decision and Control, 1985.

[22] D. Kubus, T. Kroger, and F. M. Wahl, "On-line rigid object recognition and pose estimation based on inertial parameters," in Proceedings of Intelligent Robots and Systems, 2007.

[23] A. Bicchi, J. K. Salisbury, and D. L. Brock, "Contact sensing from force measurement," The International Journal of Robotics Research, vol. 12, pp. 249-262, 1993.

[24] S. J. Lederman and R. L. Klatzky, "Haptic identification of common objects: Effects of constraining the manual exploration process," Perception \& Psychophysics, vol. 66, no. 4, pp. 618 - 628, 2004.

[25] ATI. F/t sensor: Nano17. [Online]. Available: http://www.ati-ia. com/products $/ \mathrm{ft} / \mathrm{ft} \_$models.aspx?id=Nano17

[26] D. W. Eggert, A. Lorusso, and R. B. Fisher, "Estimating 3-d rigid body transformations: a comparison of four major algorithms," Machine Vision and Applications, vol. 9, pp. 272 - 290, 1997.

[27] P. Space. Motion capture system. [Online]. Available: http: //www.phasespace.com/

[28] C. D. Meyer, Matrix Analysis and Applied Linear Algebra. Philadelphia, PA: Society for Industrial and Applied Mathematics, 2000. 